\title{
Resonant subharmonic absorption and second-harmonic generation by a fluctuating nonlinear oscillator
}

\author{
M. I. Dykman, ${ }^{1}$ D. G. Luchinsky,,${ }^{2,}$ R. Mannella, ${ }^{3}$ P. V. E. McClintock,${ }^{2}$ S. M. Soskin,,${ }^{2, \dagger}$ and N. D. Stein ${ }^{2}$ \\ ${ }^{1}$ Department of Physics and Astronomy, Michigan State University, East Lansing, Michigan 48823 \\ ${ }^{2}$ School of Physics and Chemistry, Lancaster University, Lancaster, LA1 4YB, United Kingdom \\ ${ }^{3}$ Dipartimento di Fisica, Università di Pisa, Piazza Torricelli 2, 56100 Pisa, Italy
}

(Received 15 February 1996; revised manuscript received 8 May 1996)

\begin{abstract}
The effect of fluctuations on the nonlinear response of an underdamped oscillator to an external periodic field at a subharmonic frequency has been investigated theoretically, numerically, and with an analog electronic circuit model. The system studied has often been analyzed in nonlinear optics in the context of twophoton absorption and second-harmonic generation. We consider its nonlinear spectroscopy. Its resonant nonlinear response is described over a broad range of the fluctuation intensities. It is shown that the fluctuation intensity can be used to "tune" the oscillator so as to maximize the nonlinear response. The dependence of the absorption cross section on the fluctuation intensity displays a clearly resolved maximum. If the eigenfrequency of the oscillator is a nonmonotonic function of its energy, the signal at the second harmonic displays a resonant peak at one of two different frequencies, depending on the noise intensity. [S1063-651X(96)10308-1]
\end{abstract}

PACS number(s): $05.40+\mathrm{j}, 05.20 . \mathrm{Dd}$

\section{INTRODUCTION}

The resonant response of a nonlinear system to periodic forcing at a subharmonic frequency is studied in several areas of physics. For a nonlinear oscillator this problem has been investigated in detail in mechanics [1]. A nonlinear oscillator also constitutes a simple and useful model that enables the susceptibility to be calculated, and is therefore of major interest for nonlinear optics [2]. The susceptibility is often analyzed with account taken of damping of the oscillator, but with a neglect of fluctuations. However, the coupling to a thermal bath that gives rise to the damping must also result in the occurrence of fluctuations. Additionally, fluctuations may result from an external noise driving the oscillator.

The role of fluctuations is particularly important for the resonant response of an underdamped oscillator. The eigenfrequency of the vibrations of a nonlinear oscillator $\omega(E)$ depends on the oscillator energy $E$ (or, equivalently, on the vibration amplitude). For a fluctuating oscillator the energy varies in time, creating a distribution over $E$ : in the case of thermal fluctuations arising from a bath at temperature $T$, this distribution is of the form $Z^{-1} \exp (-E / T)$. As a result, the eigenfrequency $\omega(E)$ fluctuates as well, with two important consequences: (i) a corresponding frequency distribution is formed, i.e., the oscillator frequency is smeared; and (ii) the mean frequency $\langle\omega(E)\rangle$ differs from the frequency of small-amplitude vibrations $\omega(0)$ (where we measure the energy relative to its value at the equilibrium position of the oscillator). Therefore, on the one hand, fluctuations may be

\footnotetext{
*Permanent address: Research Institute for Metrological Service, 117965 Moscow, Russia.

Permanent address: Institute of Semiconductors, Ukrainian Academy of Sciences, Kiev, Ukraine.
}

used to "tune" the system to a resonance with the frequency of the external force or its overtones. On the other hand, with increasing fluctuation intensity the resonance itself is smeared out. The latter occurs when the frequency smearing $\delta \omega$ due to the fluctuations exceeds the uncertainty in the vibration frequency due to finite damping. For small fluctuation intensity the frequency smearing is given by $\delta \omega=\left|\omega^{\prime}(0)\right|\langle E\rangle$, where $\omega^{\prime}(E) \equiv d \omega(E) / d E$.

The effects of classical and quantum fluctuations on the linear response of a nonlinear oscillator have been investigated in considerable detail (cf. [3-13]; see also [14] for reviews). The problem is immediately related to the infrared absorption spectra of localized vibrations in solids [15], and also to problems of acoustics and of engineering structures. An advantageous feature of using a fluctuating oscillator as a model is that, in case of an underdamped oscillator, it is asymptotically exactly solvable: although the nonlinearity of the oscillator is by no means small, a theoretical analysis of the resonant response can be fulfilled in closed form [7] (see also $[10,13])$. The nonlinear response of the oscillator to a nearly resonant field has also been analyzed in considerable detail, and the results discussed in the context of optical bistability and phase conjugation [16], transmission of high$Q$ optical and microwave cavities [17], cyclotron resonance of an electron in a Penning trap [18], and underdamped vibrations of ions trapped in a potential well below the surface of liquid helium [19].

In the present paper we investigate the effect of fluctuations in an underdamped oscillator on its resonant nonlinear response to driving at a subharmonic frequency. We concentrate on the case of driving at a frequency $\Omega$ close to $\frac{1}{2}$ $\omega(E)$ for energies $E$ that lie within the width of the distribution over $E$. In this case, the nonlinear resonant absorption, in terms of nonlinear optics, corresponds to two-photon absorption (TPA), whereas the occurrence of forced vibrations of the oscillator at frequency $2 \Omega$ corresponds to 
second-harmonic generation (SHG). TPA and SHG have been recently recognized as particularly advantageous for investigating systems with broken inversion symmetry, like interfaces between isotropic media (liquid-vapor interface, etc) [20-22]. Often the immediate object of investigation is nonlinear vibrations at the interface (as in references cited above), and in many cases such vibrations are underdamped. Understanding the spectroscopy of TPA and SHG, and the effects of fluctuations on the corresponding spectra, are extremely important in this context.

Based on the arguments given above one would expect that, in the appropriate range of $\Omega$, it would be possible to increase the nonlinear response by increasing the fluctuation intensity and thus "tuning" the oscillator to resonance, but that, with further increase in the fluctuation intensity, the response would decrease because of the broadening of the resonance due to frequency smearing. Such a behavior of the response as a function of noise intensity has recently attracted much attention in the context of stochastic resonance [23]. Stochastic resonance is most often considered for bistable systems, but there is no reason a priori to suppose that it need be restricted to such systems. Indeed, a noiseinduced increase in linear response has been demonstrated in analog experiments for a resonantly driven monostable underdamped oscillator [24].

If the eigenfrequency of an oscillator is a nonmonotonic function of its energy, an increase of noise intensity may result in a narrowing of the peak of the resonant absorption [12] and the occurrence, for very small damping and within a certain range of noise intensities, of an additional peak at the extremal frequency $\omega_{e}$ [25]. One would expect a similar peak to occur in the spectrum of nonlinear absorption.

In Sec. II we discuss the model and provide an analytical theory of the resonant nonlinear response of an underdamped nonlinear oscillator. The theory is valid for weak noise; it is perturbative in the amplitude of the driving field, but it is nonperturbative in the oscillator nonlinearity. In Sec. III we describe the analog simulator. In Sec. IV we present experimental results and compare them with the theory. Section V contains concluding remarks. In Appendix A we analyze the resonant nonlinear response for noise-induced frequency smearing $\delta \omega$ greatly exceeding the oscillator damping. The method of moments for the nonresonant linear response of a nonlinear oscillator in the case of weak noise intensities is described in Appendix B.

\section{THEORY OF AN UNDERDAMPED OSCILLATOR DRIVEN AT A SUBHARMONIC FREQUENCY}

\section{A. Model}

We will analyze fluctuation effects for the simplest model that possesses a resonant second-order susceptibility: a noncentrosymmetric Duffing oscillator. This is the model that, in the absence of fluctuations, has traditionally been considered in connection with a variety of problems in nonlinear optics [2]. We will limit ourselves to a phenomenological analysis in which fluctuations are induced by an externally applied noise $f(t)$. The noise will be assumed to be white and Gaussian.

The equation of motion of the oscillator is of the form

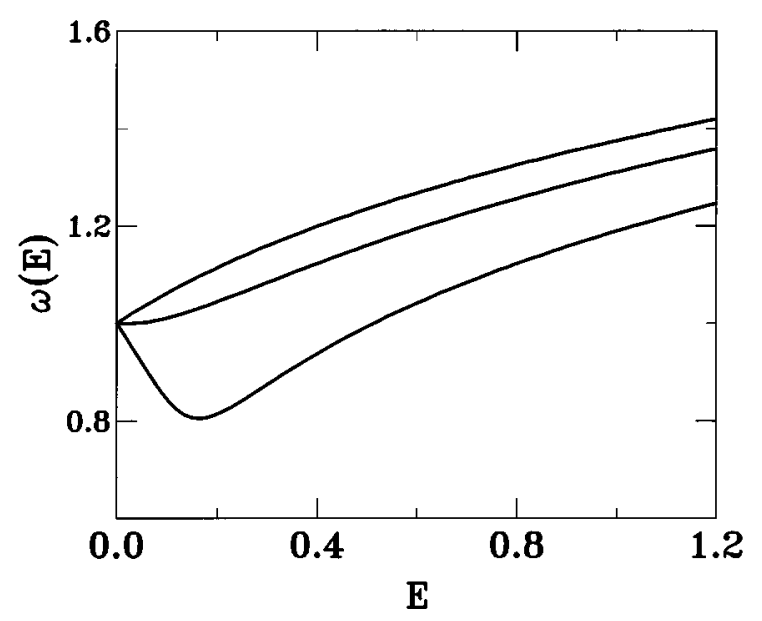

FIG. 1. The dependence of the oscillation eigenfrequency $\omega(E)$ on the energy $E$ of the oscillator (1), measured from the bottom of the potential well, for different values of the parameter $\beta$ with $\gamma=\omega_{0}=1$. The values of $\beta$ corresponding to the three curves, from bottom to top, were 1.666, 1.000, and 0.300. All quantities are in dimensionless units.

$$
\begin{gathered}
\ddot{q}+2 \Gamma \dot{q}+\omega_{0}^{2} q+\beta q^{2}+\gamma q^{3}=F \cos \Omega t+f(t), \\
\langle f(t)\rangle=0, \quad\left\langle f(t) f\left(t^{\prime}\right)\right\rangle=4 \Gamma T \delta\left(t-t^{\prime}\right) .
\end{gathered}
$$

We assume the oscillator to be monostable, which is the case provided $\gamma>0, \beta^{2}<4 \omega_{0}^{2} \gamma$. The energy dependence of the eigenfrequency of the vibrations $\omega(E)$ (the dispersion curve) is shown for $\gamma=\omega_{0}=1$ in Fig. 1 . For $\beta^{2} / \gamma \omega_{0}^{2}>9 / 10$ the function $\omega(E)$ is nonmonotonic, with a minimum at a frequency $\omega_{e}$. We further assume that the friction coefficient $\Gamma$ is small compared both to the frequency of smallamplitude vibrations $\omega_{0} \equiv \omega(0)$ and to the frequency detuning of a nonresonant periodic force $F \cos \Omega t$,

$$
\Gamma \ll \omega(E), \Omega,|\omega(E)-\Omega|,
$$

for $E \leqq T$. The characteristic noise intensity $T$ in (1) corresponds to temperature if both the noise and the friction force $-2 \Gamma \dot{q}$ originate from the coupling of the oscillator to a thermal bath.

We note that many of the results discussed below will actually apply to a much broader class of systems than white-noise-driven systems with a friction force proportional to the instantaneous value of the velocity. What is important is that the effects of retardation on the slowly varying (on a time scale $\sim \omega_{0}^{-1}$ ) amplitude and phase of the oscillator be small [14].

In the analysis of the resonant nonlinear response, we assume that the doubled frequency of the force, $2 \Omega$, is close to the band of frequencies of thermally excited vibrations of the oscillator,

$$
|2 \Omega-\omega(E)| \ll \omega(E) \text { for } E \lesssim T .
$$

Theoretical formulations need to be somewhat different for different ranges of noise intensity $T$. We shall concentrate in this section on the case where $T$ is moderately small, 
so that the nonlinear terms $\frac{1}{3} \beta q^{3}+\frac{1}{4} \gamma q^{4}$ in the potential energy of the oscillator are small compared to the harmonic term $\frac{1}{2} \omega_{0}^{2} q^{2}$ :

$$
T \ll \omega_{0}^{4} / \gamma, \quad \omega_{0}^{6} / \beta^{2} .
$$

The major effect of noise in range (3) is smearing of the frequency of the oscillator. Although the frequency straggling $\delta \omega$ remains relatively small, $\delta \omega \equiv\left|\omega(T)-\omega_{0}\right| \ll \omega_{0}$, and so also are the amplitudes of noise-induced vibrations at the overtones of the eigenfrequencies $\omega(E)$, the straggling $\delta \omega$ may substantially exceed the frequency uncertainty $\Gamma$ due to damping. Therefore, in the sense that the ratio $\delta \omega / \Gamma$ may still be large, the nonlinearity and fluctuations cannot be assumed to be small. Consequently, the resonant nonlinear response is qualitatively different from that in the neglect of fluctuational smearing of the frequency. The case where the noise intensity is not limited by (3) is considered in Appendix A; in the range $T \gtrsim \omega_{0}^{4} / \gamma, \omega_{0}^{6} / \beta^{2}$, one may neglect frequency smearing due to dissipation, which in many respects simplifies the problem.

\section{B. Quasilinear response}

For small amplitudes of the driving force and for moderately weak noise intensities (3), the analysis of the resonant nonlinear response of the oscillator may be done in two steps familiar from [1]: (i) one first ignores the oscillator nonlinearity, so that a sinusoidal external force results in nonresonant vibrations at the force frequency $\Omega$ (the perturbation parameter is the force amplitude $F$, weighted with nonlinearity constants); and (ii) in the next iteration one substitutes the corresponding oscillating term in the oscillator coordinate into the nonlinear terms in the restoring force of the oscillator. These terms then oscillate at the overtones of $\Omega$, and act as an effective force that drives the oscillator. The resonant linear response to this force may then be analyzed. In this way, for an oscillator with a non-weak nonlinearity, a complicated problem of resonant nonlinear response may be reduced to the already solved problem of resonant linear response.

For moderately small noise intensities (3) and to lowest order in the force amplitude $F$, nonresonant forced vibrations may be described in the harmonic approximation,

$$
\begin{gathered}
q^{(1)}(t) \approx \frac{F}{\omega_{0}^{2}-\Omega^{2}} \cos \left(\Omega t+\phi^{(1)}\right), \\
\phi^{(1)} \approx-2 \Gamma \Omega /\left(\omega_{0}^{2}-\Omega^{2}\right) .
\end{gathered}
$$

The phase shift $\phi^{(1)}$ allows for a weak linear absorption by the oscillator. The absorption coefficient $\kappa$ is defined as the ratio of the average energy absorbed per unit time to $F^{2}$. In the limit of small field $\kappa=\kappa^{(1)}$, where $\kappa^{(1)}$ is the coefficient of linear nonresonant absorption and, with account taken of (4),

$$
\begin{gathered}
\kappa=F^{-2} \overline{\langle\dot{q}(t) F \cos \Omega t\rangle}, \quad \kappa \rightarrow \kappa^{(1)} \text { for } F \rightarrow 0, \\
\kappa^{(1)}=\Gamma \Omega^{2} /\left(\omega_{0}^{2}-\Omega^{2}\right)^{2}
\end{gathered}
$$

(the overbar denotes averaging over the period $2 \pi / \Omega$ ).
The equation of motion for the vibrations at frequency $2 \Omega$ in the range of moderately small noise intensities (3) can be obtained by seeking the solution of Eq. (1) in the form $q(t) \approx q^{(1)}(t)+q^{(2)}(t)$. The equation for $q^{(2)}(t)$ may be written as

$$
\begin{aligned}
\ddot{q}^{(2)} & +2 \Gamma \dot{q}^{(2)}+\omega_{0}^{2} q^{(2)}+\beta\left[q^{(2)}\right]^{2}+\gamma\left[q^{(2)}\right]^{3} \\
& \approx f(t)-\frac{F^{2}}{2\left(\omega_{0}^{2}-\Omega^{2}\right)^{2}} \beta\left[\cos \left(2 \Omega t+2 \phi^{(1)}\right)+1\right] .
\end{aligned}
$$

We have neglected terms of higher order in $F$ as well as terms $\propto \beta F q^{(2)}, \gamma F\left[q^{(2)}\right]^{2}, \gamma F^{2} q^{(2)}$. These terms contain, in addition to $F$, a small [in range (3)] factor proportional to the amplitude of fluctuational vibrations. (The term $\propto \gamma F^{2} q^{(2)}$ describes the frequency shift $\propto \gamma F^{2}$, which is similar to the frequency shift in the dynamical Stark effect. It could easily be incorporated; but we have chosen not to do so because it may be assumed small compared to the frequency smearing due to nonlinearity and damping.) The nonresonant timeindependent term on the right-hand side of (6) is retained for the sake of clarity; it gives rise to a shift of the equilibrium position of the nonlinear oscillator due to periodic driving.

Equation (6) is the equation of motion of a nearly resonantly driven nonlinear oscillator with coordinate $q^{(2)}$. For small $F$ the response of the oscillator to the force $\propto \beta F^{2}$ can be described by linear response theory [26]:

$$
\begin{aligned}
\left\langle q^{(2)}\right\rangle \approx & -\frac{\beta F^{2}}{2 \omega_{0}^{2}\left(\omega_{0}^{2}-\Omega^{2}\right)^{2}} \\
& -\frac{\beta F^{2}}{4\left(\omega_{0}^{2}-\Omega^{2}\right)^{2}}\left[\chi(2 \Omega) e^{-2 i\left(\Omega t+\phi^{(1)}\right)}\right. \\
& \left.+\chi^{*}(2 \Omega) e^{2 i\left(\Omega t+\phi^{(1)}\right)}\right],
\end{aligned}
$$

where $\chi(2 \Omega)$ is the susceptibility of the oscillator at $2 \Omega$.

In the frequency range (2) the susceptibility $\chi(2 \Omega)$ is resonantly large. In the absence of noise, $\chi(2 \Omega) \approx\left(4 \Omega^{2}-\omega_{0}^{2}-4 i \Gamma \Omega\right)^{-1}$. The imaginary part of the susceptibility $\chi^{\prime \prime}(2 \Omega)$ for a finite noise intensity [but limited by (3)], and for $\left|2 \Omega-\omega_{0}\right| \ll \Omega$, was evaluated in our former work [7]. A complete analysis of the susceptibility $\chi(2 \Omega) \equiv \chi^{\prime}(2 \Omega)+i \chi^{\prime \prime}(2 \Omega)$ is presented in Sec. II C.

In terms of nonlinear optics, the onset of vibrations at twice the frequency of the driving force corresponds to second-harmonic generation (SHG). Equation (7) fully describes resonant SHG in an underdamped fluctuating nonlinear oscillator.

Equation (7) also makes it possible to analyze resonant absorption at frequency $2 \Omega$, i.e., two-photon absorption (TPA), in the language of nonlinear optics. To do this one has to iterate Eq. (1) once more and find the term $q^{(3)}(t)$ which is of third order in $F$. This term should then be substituted into Eq. (5) for the absorption coefficient $\kappa$. The resulting overall expression for $\kappa$ allows both for nonresonant linear (in $F$ ) absorption due to oscillator damping, and for a resonant nonlinear (in $F$ ) absorption. It takes the form 


$$
\begin{gathered}
\kappa \approx \kappa^{(1)}+\kappa^{(2)}, \\
\kappa^{(2)}=\frac{\Omega}{4\left(\omega_{0}^{2}-\Omega^{2}\right)^{2}}\left(\frac{\beta F}{\omega_{0}^{2}-\Omega^{2}}\right)^{2} \chi^{\prime \prime}(2 \Omega),
\end{gathered}
$$

where $\kappa^{(1)}$ and $\kappa^{(2)}$ are the coefficients of linear and nonlinear absorption, respectively. The coefficient $\kappa^{(1)}$ for small noise intensities is given by (5).

It follows from (8) that, in range (3), TPA as a function of frequency and temperature should display a behavior similar to that displayed by linear absorption of a nonlinear oscillator, which is described by the imaginary part of the susceptibility $\chi^{\prime \prime}(2 \Omega)$. We note that the proportionality factor between $\kappa^{(2)}$ and $\chi^{\prime \prime}(2 \Omega)$ is the squared coefficient of the cubic nonlinearity $\beta$, in agreement with the well-known fact that TPA occurs in noncentrosymmetric systems.

\section{Resonant susceptibility}

Equations (7) and (8) relate the intensity of the second harmonic signal (SHS) and the coefficient of nonlinear absorption $\kappa^{(2)}$ to the resonant linear susceptibility of the oscillator $\chi(2 \Omega)$ at frequency $2 \Omega$. Theoretical and experimental results on the evolution with noise intensity of the imaginary part of the susceptibility $\chi^{\prime \prime}(\omega)$ have clearly demonstrated [7,10-12,24] that, in an underdamped system, even comparatively small changes in the noise intensity can strongly affect linear resonant absorption.

Complete analysis of the susceptibility is facilitated by the fact that, in the absence of the periodic force, the system investigated is effectively in thermal equilibrium at temperature $T$ which is characterized by the noise intensity. For thermal equilibrium systems the real and imaginary parts of the susceptibility $\chi(\omega)$ are related to each other via KramersKronig relations, and both can be expressed in terms of the spectral density of fluctuations of the oscillator $Q^{(0)}(\omega)$ [26]:

$$
\begin{gathered}
\chi^{\prime \prime}(\omega)=\frac{\pi \omega}{T} Q^{(0)}(\omega) \\
\chi^{\prime}(\omega)=\frac{2}{T} \mathrm{P} \int_{0}^{\infty} d \omega_{1} Q^{(0)}\left(\omega_{1}\right) \frac{\omega_{1}^{2}}{\omega_{1}^{2}-\omega^{2}}, \\
Q^{(0)}(\omega)=\frac{1}{2 \pi} \int_{-\infty}^{\infty} d t e^{i \omega t}\langle q(t) q(0)\rangle
\end{gathered}
$$

where $\mathrm{P}$ implies the Cauchy principal value and $T$ is the temperature.

The quantity $Q^{(0)}(\omega)$ for an underdamped nonlinear oscillator has been investigated analytically in considerable detail for the range of moderately small noise intensities (3) [7], and numerically and experimentally in the general case [12].

In Figs. 2 and 3 we show theoretical curves for the squared absolute value of the susceptibility $|\chi(\omega)|^{2}$ as a function of the noise intensity $T$ for five values of $\omega=2 \Omega$, and as a function of $\omega$ for three values of $T$, respectively. In Fig. 4 we show the dependence on $T$ of the real and imaginary parts of $\chi(\omega)$. All curves have been obtained using the

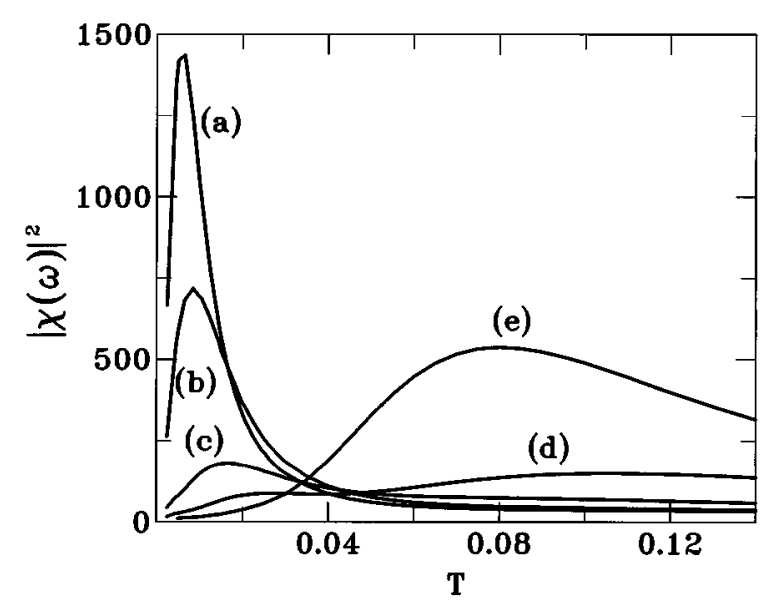

FIG. 2. Dependence of the susceptibility on noise intensity, for $\beta=1.666$. The curves represent the calculated $|\chi(\omega)|^{2}$ at the second harmonic $(\omega=2 \Omega)$ of the field frequency $\Omega$. The frequencies were (a) $\Omega / \omega_{0}=0.490$, (b) 0.483 , (c) 0.463 , (d) 0.440 , and (e) 0.415 .

algorithm of [12] and relations (9), and the parameters were taken to be the same as in the experimentally investigated system (see Sec. III).

It is clear from Figs. 2 and 4 that, both for $\omega \approx \omega_{0}$ and $\omega \approx \omega_{e}$, the functions $\chi^{\prime}(\omega), \chi^{\prime \prime}(\omega)$, and $|\chi(\omega)|^{2}$ display nonmonotonic dependences on $T$. The peaks in $\chi^{\prime \prime}(\omega)$ and $|\chi(\omega)|^{2}$ vs $T$ are most pronounced for $\omega \approx \omega_{0}$. This is due to the fact that noise-induced "tuning", of an underdamped oscillator to a given frequency is most effective for small $T$, where the fluctuational smearing $\delta \omega$ of the oscillator frequency is small and the oscillator may display a strongly resonant response.

The spectral shape and position of the maximum of the narrow peak of the function $\chi^{\prime \prime}(\omega)$, and their variation with temperature for $\delta \omega \lesssim \Gamma$, have been described in [7]. With increasing noise intensity the position of the maximum, $\omega_{m}$ shifts in the direction determined by the sign of $d \omega(E) / d E(E \rightarrow 0)$, and the peak is broadened and becomes non-Lorentzian. As a result the absorption can decrease or

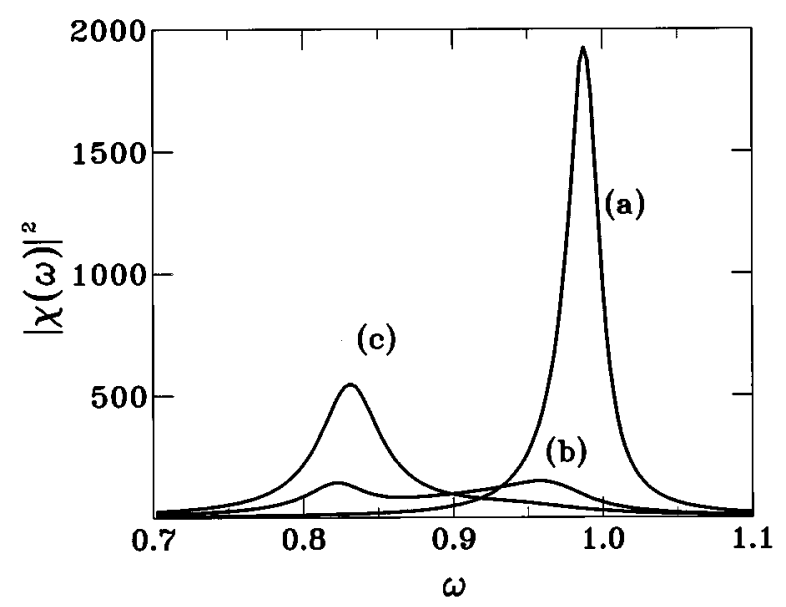

FIG. 3. Frequency dependence of $|\chi(\omega)|^{2}$ for different values of the noise intensity: (a) $T=0.0065$, (b) 0.035 , and (c) 0.080 . 


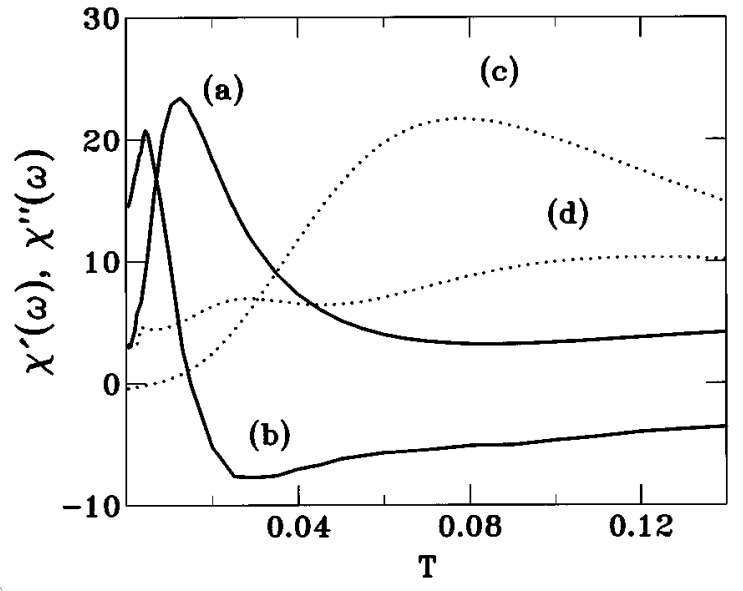

FIG. 4. Dependence on noise intensity $T$ of the susceptibility at the second harmonic $(\omega=2 \Omega)$ for two different frequencies: $\Omega / \omega_{0}=0.483$ (solid curves) and 0.415 (dotted curves). The real parts $\chi^{\prime}$ are shown by (b) and (d), and the imaginary parts $\chi^{\prime \prime}$ by (a) and $(\mathrm{c})$.

increase (within a limited range of noise intensities) depending on the frequency of the external field.

It is seen from Fig. 3 that, for higher $T$, the peak of $|\chi(\omega)|^{2}$ at $\omega \approx \omega_{0}$ is broadened, and for yet higher $T$ the dominating peak in $|\chi(\omega)|^{2}$ is the one at $\omega_{e}$ which is due to the singularity in the density of vibrational states of the oscillator $[12,25]$.

\section{Nonresonant susceptibilities}

Although the nonlinear absorption that we investigate is resonant, it is comparatively small for small amplitudes of the driving force $F$. Therefore, in the analysis of the effect of noise on the total (linear + nonlinear) absorption of the oscillator, it is necessary to take into account not only noiseinduced changes in nonlinear absorption, but also noiseinduced corrections to linear nonresonant absorption. These corrections are obtained in Appendix B by solving the Fokker-Plank equation. Alternatively, they can be found by a perturbation method applied directly to the Langevin equation (1).

With account taken of corrections linear in $T$, the susceptibility $\chi(\Omega)$ is of the form

$$
\begin{gathered}
\chi(\Omega) \approx \chi^{(0)}(\Omega)\left(1+A \frac{\gamma T}{\omega_{0}^{2}}+B \frac{\beta^{2} T}{\omega_{0}^{4}}\right), \\
A=-\frac{3}{\omega_{0}^{2}-\Omega^{2}}\left(1+i \frac{2 \Gamma \Omega}{\omega_{0}^{2}-\Omega^{2}}\right), \\
B=\frac{2\left(6 \omega_{0}^{2}-\Omega^{2}\right)}{\left(4 \omega_{0}^{2}-\Omega^{2}\right)\left(\omega_{0}^{2}-\Omega^{2}\right)} \\
+\frac{4 i \Gamma}{\Omega} \frac{8 \omega_{0}^{6}+18 \omega_{0}^{4} \Omega^{2}-12 \omega_{0}^{2} \Omega^{4}+\Omega^{6}}{\left(4 \omega_{0}^{2}-\Omega^{2}\right)^{2}\left(\omega_{0}^{2}-\Omega^{2}\right)^{2}} .
\end{gathered}
$$

It is clear from Eqs. (4) and (5) that, for small absorption, the absorption coefficient is proportional to the phase shift between the signal $q(t)$ and the force. An advantageous feature

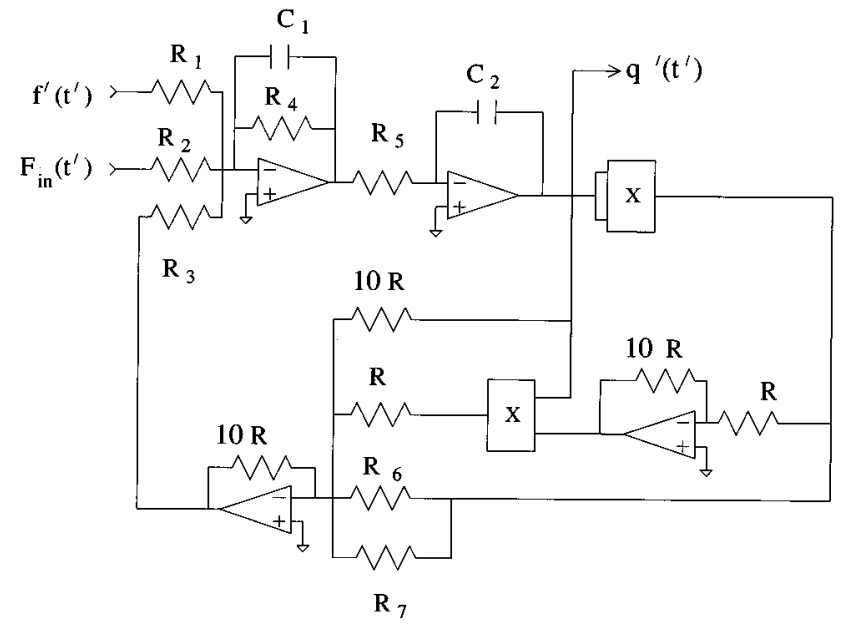

FIG. 5. Block diagram of the electronic circuit used to model the system (1) in the experiments. The component values were chosen such that $\gamma=1.00, \beta=1.67, \omega_{0}=1.00$, and the damping constant $\Gamma=0.0061$.

of the phase shift is that it can be easily determined by experiment. At the same time, it is given just by the negative of the imaginary part of the logarithm of $\chi$, and its $T$-dependent component is determined by the imaginary part of the correction $\propto T$ in (10).

\section{ANALOG ELECTRONIC MODEL AND NUMERICAL ALGORITHMS}

The effects of noise on resonant SHG and TPA have been investigated in analog electronic experiments. The analog model of (1) was of a standard design, constructed on the basis of the principles described in detail elsewhere [27], using operational amplifiers, multipliers, and other standard analog components to perform the required mathematical operations of addition, subtraction, multiplication, division, integration, etc. A block diagram of the circuit is shown in Fig. 5. Here $F_{\text {in }}\left(t^{\prime}\right)=F^{\prime} \cos \Omega^{\prime} t^{\prime}$ and $f^{\prime}\left(t^{\prime}\right)$, are respectively, a signal and an external noise applied to an underdamped nonlinear oscillator. $F^{\prime}$ is the amplitude of the signal in volts, $f^{\prime}\left(t^{\prime}\right)$ is the value of the noise voltage applied to the circuit, and $\Omega^{\prime}$ and $t^{\prime}$ are the real frequency and time.

The differential equation for the voltage $x$ in the circuit is of the form

$$
\begin{aligned}
& R_{3} C_{1} R_{5} C_{2} \frac{d^{2} x}{d t^{\prime 2}}+\frac{R_{3}}{R_{4}} R_{5} C_{2} \frac{d x}{d t^{\prime}}+x+R\left(1 / R_{6}+1 / R_{7}\right) x^{2} \\
& \quad+x^{3}+\frac{R_{3}}{R_{2}} F^{\prime} \cos \Omega^{\prime} t^{\prime}+\frac{R_{3}}{R_{1}} f^{\prime}\left(t^{\prime}\right) \\
& =0
\end{aligned}
$$

where we have chosen

$$
\begin{gathered}
R=R_{6}=10 \mathrm{k} \Omega, \quad R_{1}=R_{2}=100 \mathrm{k} \Omega, \\
R_{3}=11 \mathrm{k} \Omega, \quad R_{5}=22 \mathrm{k} \Omega, \quad R_{4}=1.1 \mathrm{M} \Omega, \\
R_{7}=15 \mathrm{k} \Omega, \quad C_{1}=C_{2}=20 \mathrm{nF} .
\end{gathered}
$$


On introducing the time constant $\tau^{\prime}=R_{5} C_{2}=2 R_{3} C_{1}$ and damping constant $\Gamma^{\prime}=R_{3} / R_{4}$, Eq. (11) reduces to

$$
\begin{aligned}
\tau^{\prime 2} \ddot{x} & +2 \Gamma^{\prime} \tau^{\prime} \dot{x}+2\left(\omega_{0}^{2} x+\beta x^{2}+\gamma x^{3}\right) \\
& =-0.22 F^{\prime} \cos \Omega^{\prime} t^{\prime}-0.22 f^{\prime}\left(t^{\prime}\right)
\end{aligned}
$$

where parameters are readily related to those in model (1) by means of the scaling relations

$$
\begin{gathered}
\tau=\frac{\tau^{\prime}}{\sqrt{2}}, \quad t=\frac{t^{\prime}}{\tau}, \quad \Omega=\Omega^{\prime} \tau, \quad \Gamma=\frac{\Gamma^{\prime}}{\sqrt{2}}, \\
\gamma=1.0, \quad \beta=1.666, \quad \omega_{0}=1.0, \quad F=-0.11 F^{\prime}, \\
f(t)=-0.11 f^{\prime}\left(t^{\prime}\right) .
\end{gathered}
$$

The nominal value of $\Gamma$ was 0.0071 . However, for such small damping, the actual value usually differs from the nominal one due to the effects of stray capacitances and resistances in the circuit. In the present case, the actual value, measured experimentally by two independent methods [28] was found to be $\Gamma=0.0061$. For the chosen circuit parameters the variation of the oscillator's eigenfrequency with energy had a minimum at $E_{m}=0.1635$, as shown in Fig. 1 (the lowest curve).

The circuit model was driven by noise from a feedback shift-register noise generator and by a sinusoidal periodic force from a Hewlett-Packard Model 3325B frequency synthesizer. The response of the circuit to the stochastic and periodic forces was analysed with the aid of a Nicolet 1080 digital data processor.

The intensity of the second harmonic of the signal was measured directly from the ensemble-averaged signal in the circuit $\langle x(t)\rangle$ at frequency $2 \Omega$. It follows from (7) that this intensity divided by $\beta^{2} F^{4} / 16\left(\omega_{0}^{2}-\Omega^{2}\right)^{4}$ gives us the value of the susceptibility $|\chi(2 \Omega)|^{2}$, provided $F$ is small ( $F=0.0176$ in our experiment).

The two-photon absorption coefficient was determined from measurements of the phase shift $\phi$ (relative to the driving force) of the ensemble-averaged signal $\langle x(t)\rangle$ at frequency $\Omega$. In the range of moderately weak noise intensities (3) the expression for the phase shift can be obtained in a way similar to that used to derive Eq. (8) for the absorption coefficient. In the limit of weak absorption the phase shift is given by a sum of the contributions $\phi^{(1)}$ and $\phi^{(2)}$ that correspond, respectively, to linear absorption and to TPA:

$$
\phi \approx \phi^{(1)}+\phi^{(2)}, \quad \phi^{(2)}=-\frac{1}{2} \frac{\beta^{2} F^{2}}{\left(\omega_{0}^{2}-\Omega^{2}\right)^{3}} \chi^{\prime \prime}(2 \Omega),
$$

where the phase shift $\phi^{(1)}$ is given by Eq. (4) with the correction determined by Eq. (10).

The digital simulation was based on the algorithm described in [29]. In all cases we used an integration time step of order $10^{-4}$. For measurement of the spectral densities of fluctuations, we averaged typically over 20000 periods of the periodic forcing, or for an equivalent total time in the absence of the periodic force.

For measurement of the phase lag as a function of noise intensity in the presence of the periodic forcing, a much longer total integration time was used: note that the integration time step itself places a lower limit on the precision of the phase measurements (see above) of $\approx 2 \times 10^{-4} \mathrm{rad}$. The number of averages included for each value of noise intensity was typically 840000 periods of the periodic force. The quoted error on the phase lag from the digital simulations was estimated by means of a $\chi^{2}$ likelihood approach: the 840000 averages were divided into 42 statistically independent samples (of 20000 averages each); the "average", phase lag was determined for each sample, and, from these 42 independent measurements of the phase lag, the bona fide phase lag corresponding to the given noise intensity and its error (taken as one standard deviation in the $\chi^{2}$ ) was computed.

For the case when periodic forcing was absent, given that the damping $\Gamma$ was typically very small so that the energy $E$ (see introduction) was changing slowly, it was also possible to integrate the equations of motion using a quasisymplectic scheme: some symplectic schemes are discussed in [30]. For the present case, the scheme used reads

$$
\begin{gathered}
q^{*}=q_{t}+\frac{h}{2} p_{t}, \\
p_{t+h}=\frac{1}{1+\Gamma h}\left[(1-\Gamma h) p_{t}+h F\left(q^{*}\right)+\sqrt{4 \Gamma T h} w\right], \\
q_{t+h}=q^{*}+\frac{h}{2} p_{t+h} .
\end{gathered}
$$

where $q_{a} \equiv q(t=a), p_{a} \equiv \dot{q}(t=a)$, etc., $h$ is the integration time step, $F(q)$ is the deterministic force, and $w$ is a random Gaussian variable with zero average and a standard deviation of unity.

The spectral densities obtained using the algorithm of Eq. (14) coincided with those obtained using the algorithm of [29].

\section{EXPERIMENTAL RESULTS AND DISCUSSION}

\section{A. Second harmonic generation}

Measurements of the intensity of the signal at the second harmonic of the forcing frequency are shown for two frequencies by the data points in Figs. 6(a) and 6(b). In the range of force amplitudes $F$ investigated, the intensity of the second-harmonic signal (SHS) was proportional to $F^{4}$, and the data in Fig. 6 have been appropriately scaled for comparison with the relevant theoretical susceptibility curves from Fig 2. Also included in Fig. 6 are theoretical values of $|\chi|^{2}$ from (9) derived from power spectra obtained via digital simulations of the dynamics. It is evident that all the results agree well within experimental error. (Direct measurements of the linear response to a weak force at the resonant frequency $\Omega \simeq \omega_{0}$ were also found to be in good agreement with theory.) It is clear that the dependence of the SHS intensity on the noise intensity is strongly nonmonotonic, the peak of the SHS intensity being particularly tall and narrow for $\Omega \approx \frac{1}{2} \omega_{0}$. The noise-induced enhancement, defined as the ratio of the maximal intensity of the signal at a given fre- 

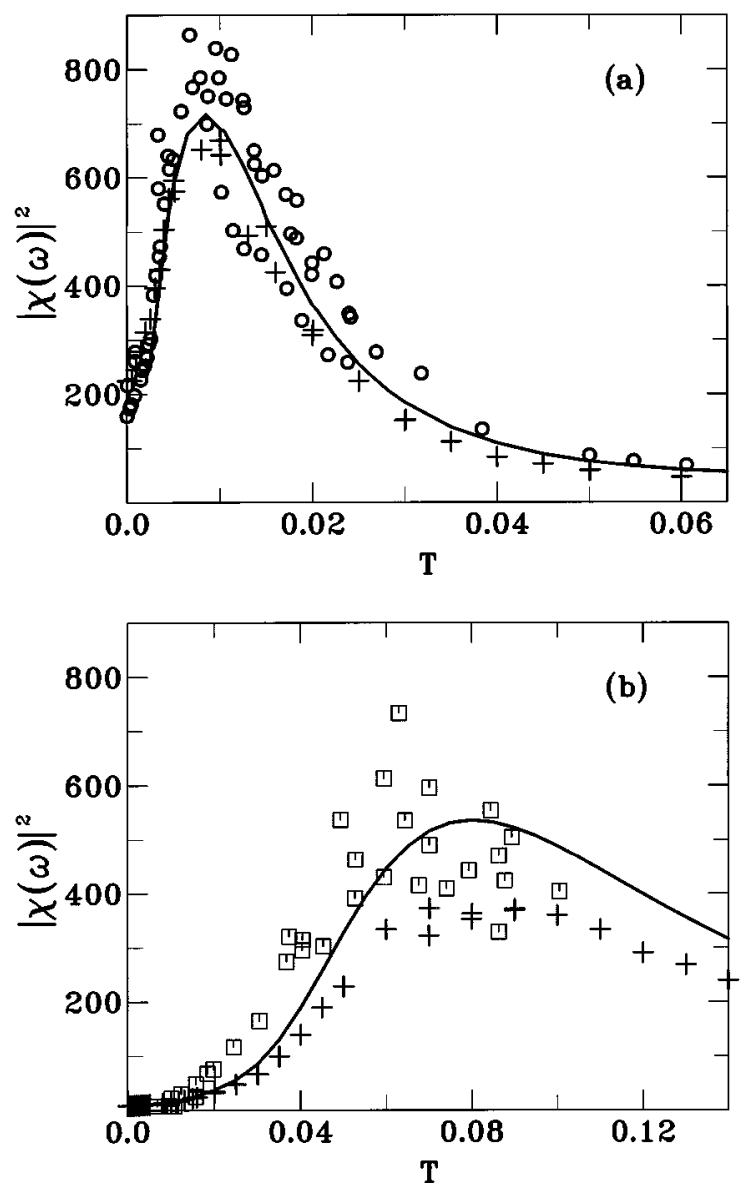

FIG. 6. Comparison of signal intensities measured for the ana$\log$ electronic model with susceptibilities calculated from (9), as a function of noise intensity $T$ in units of $\omega_{0}^{4} / \gamma$. The measured values (circles and squares) have been scaled by $\beta^{2} F^{4} / 16\left(\omega_{0}^{2}-\Omega^{2}\right)^{4}$ with $\beta=1.666$ and $F=1.76 \times 10^{-2}$ to facilitate direct comparison with the relevant theoretical curves from Fig. 2. The pluses also represent calculated susceptibilities, but using in (9) spectral densities derived from digital simulations of the dynamics. The frequencies used were (a) $\Omega / \omega_{0}=0.483$ and (b) 0.415 .

quency to the intensity for $T=0$, was found to exceed a factor of 4.5 under the experimental conditions.

To understand the temperature dependence of the SHS, it is illuminating to consider how resonance curves in the frequency domain evolve with temperature: see Fig. 3. As explained above, the increase of the SHS is due to the fact that, by increasing $T$, the system can be tuned to resonance with the frequency $2 \Omega$. The left-hand peaks in Fig. 2 occur for values of the noise intensity given, in order of magnitude, by $T \sim\left(2 \Omega-\omega_{0}\right) / \omega^{\prime}(0)$. With further increase in $T$, (i) the maximum in the frequency response shifts away from $2 \Omega$; and (ii) the half-width of the resonant peak in the susceptibility as a function of frequency increases (from the value $\Gamma$ which is determined by dissipative spectral broadening for low $T$ where $\Gamma \gg \delta \omega$, to $\delta \omega$ for higher $T$ where $\delta \omega \gg \Gamma$ ). Therefore the SHS intensity decreases, and the overall dependence of the signal on the noise intensity displays a sharp peak.

An interesting feature of the SHS intensity is that, for the case where $\omega(E)$ has a minimum, it may also display an additional peak as a function of $T$. This occurs when the frequency of the force $\Omega \approx \omega_{e} / 2$, where $\omega_{e} \equiv \omega\left(E_{e}\right)$ is the extremal value of the frequency $\omega(E)$,

$$
\omega^{\prime}\left(E_{e}\right)=0, \quad \omega^{\prime}(E) \equiv d \omega(E) / d E .
$$

The nonmonotonicity of $\omega(E)$ gives rise to a singularity in the density of vibrational states $[d \omega(E) / d E]^{-1}$. In turn, this singularity gives rise to noise-induced narrowing of the peak of $\chi^{\prime \prime}(\omega)$ with increasing noise intensity [12], and the occurrence of a characteristic zero-dispersion peak in $\chi^{\prime \prime}(\omega)$ at $\omega_{e}[25]$ for extremely low damping.

One would expect that the singularity at $\omega_{e}$ might affect the resonant nonlinear response for $\Omega \approx \frac{1}{2} \omega_{e}$, and for noise intensities $T$ of the order of $E_{e}$. It is this effect that is seen in Figs. 2 and 6.

The sharp noise-induced increase and subsequent decrease of the response with increasing noise intensity seen in Fig. 2 may be viewed as a manifestation of stochastic resonance. Two distinctive features should be emphasized in this context: (i) the system we have investigated is monostable, and the increase of the response is not related to a noiseinduced increase of the rate of switching between coexisting stable states of a bistable system as is often investigated in stochastic resonance [23]; and (ii) in contrast to [24] we observe the effect of the noise-induced increase of a nonlinear response, whereas the nonresonant linear response changes only slightly with varying noise intensity. One may say that, within a certain range of noise intensities, noise may make a system "more nonlinear," but eventually with further increase of $T$ the nonlinearity is suppressed [31].

\section{B. Nonlinear absorption}

As explained above in Sec. III, a convenient way to investigate the absorption of a periodic driving force $F \cos \Omega t$ in an analog experiment is to determine the phase shift between the ensemble-averaged signal $\langle x(t)\rangle$ at frequency $\Omega$ and the force itself. It follows from the theory [see Eqs. (8) and (13)] that the absorption coefficient and the phase shift are each made up of a sum of two components, representing linear and nonlinear contributions. In Figs. 7(a) and 8(a) we compare the measured and calculated dependences of the total phase shift $\phi$ on the noise intensity. It is clear that $|\phi|$ displays a peak as a function of $T$, that for $\Omega \approx \frac{1}{2} \omega_{0}$ this peak is comparatively narrow and sharp, and that for $\Omega \approx \frac{1}{2} \omega_{e}$ the peak, although well-resolved, is much broader. The theoretical curves (solid lines) in Figs. 7(a) and 8(a) were calculated using Eq. (13) with $\phi^{(1)}$ given by (4) with the $T$-dependent correction $-\operatorname{Im}[\ln \chi(\Omega)]$ from (10), and $\phi^{(2)}$ given by (13) with $\chi(2 \Omega)$ from (9). To evaluate the spectral density of fluctuations in (9), we used the numerical algorithm of [12].

We also carried out an additional numerical experiment in which the phase lag at the frequency of the weak periodic force was found directly, as described in Sec. III, and the spectral density of fluctuations $Q^{(0)}(\omega)$ (in the absence of the driving force) was obtained by Monte Carlo simulation of the dynamics. The resultant values of $Q^{(0)}(\omega)$ were used to evaluate the phase shift according to Eqs. (9) and (13), with $\phi^{(1)}$ given by $-\arctan \chi^{\prime \prime}(\Omega) / \chi^{\prime}(\Omega)$. In Figs. 7 and 8 these results are compared with those derived from theory 

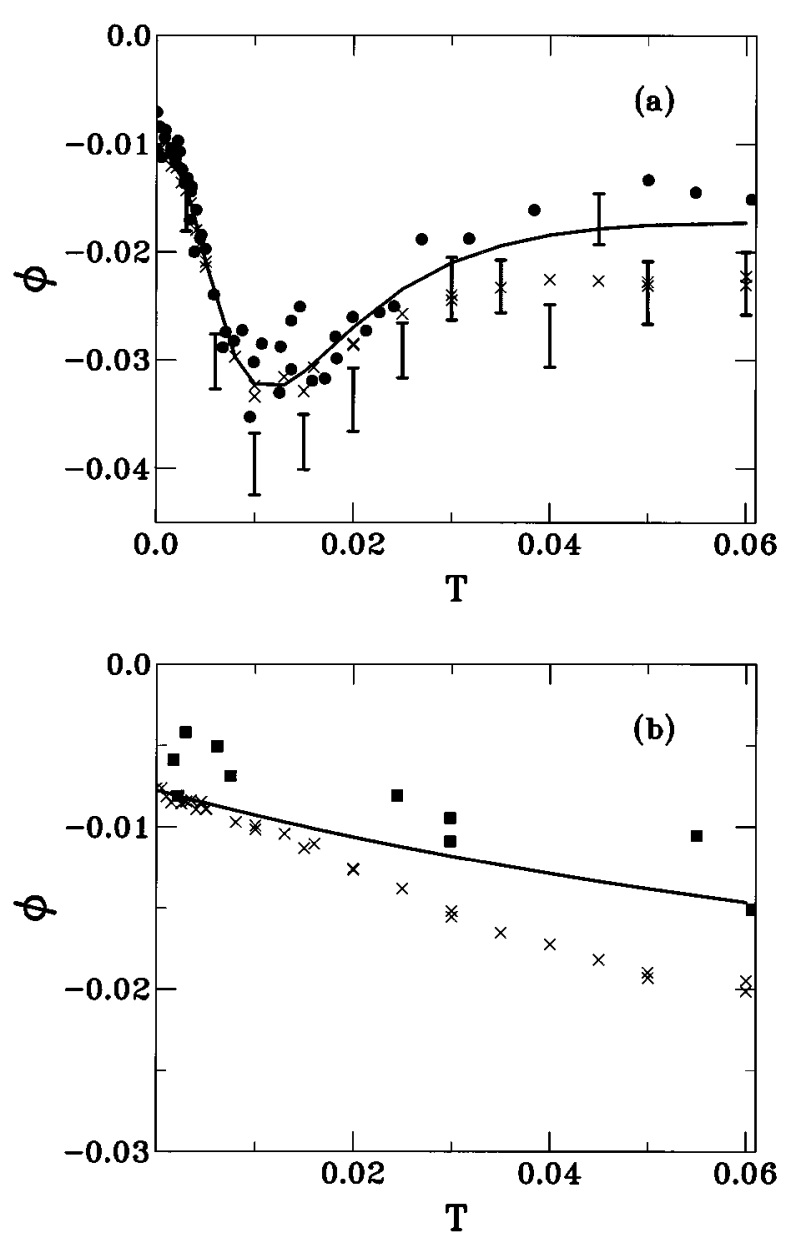

FIG. 7. (a) Dependence on noise intensity $T$ (in units of $\left.\omega_{0}^{4} / \gamma\right)$ of the phase $\phi$ of the response at the frequency $\Omega$ of the driving force for $\Omega / \omega_{0}=0.483$ and the reduced field amplitude $F=1.76 \times 10^{-2}$ measured for the analog model (solid circles). The results are compared to the theory (solid line) for $\phi$ (see text), to the values of $\phi$ obtained from computer simulations of the spectral density of fluctuations (crosses), and to direct computer simulations of $\phi$ for these parameters (bars). (b) As in (a), but without direct computer simulations and with $F=4.4 \times 10^{-3}$, i.e., for a driving force only a quarter as strong, so that $\phi \approx \phi^{(1)}$.

and from analog experiment. All the results - including those from theory, direct analog simulations, and numerical simulations, both direct and in the absence of force - are in good agreement with each other.

As discussed at the end of Sec. II, the contribution of the linear nonresonant phase shift to the total phase shift is very important when the nonlinear resonant effect is relatively small. It is especially true for the broad and relatively weak peak at $\Omega \approx \frac{1}{2} \omega_{e}$. For this reason we have measured the phase shift for very small force amplitudes when $\phi \approx \phi^{(1)}$ (see below). The temperature dependences of the measured phase shift $\phi$ and the calculated linear phase shift $\phi^{(1)}$ are shown, for two different frequencies, in Figs. 7(b) and 8(b). It can be seen that $\phi^{(1)}$, as given by (4) with the $T$-dependent correction $-\operatorname{Im}[\ln \chi(\Omega)]$ from (10), is in reasonable agreement with both the measured $\phi \approx \phi^{(1)}$ and with the values of $\phi^{(1)}=-\arctan \chi^{\prime \prime}(\Omega) / \chi^{\prime}(\Omega)$ obtained by computer simulation of $Q^{(0)}(\omega)$. (The direct computer simula-
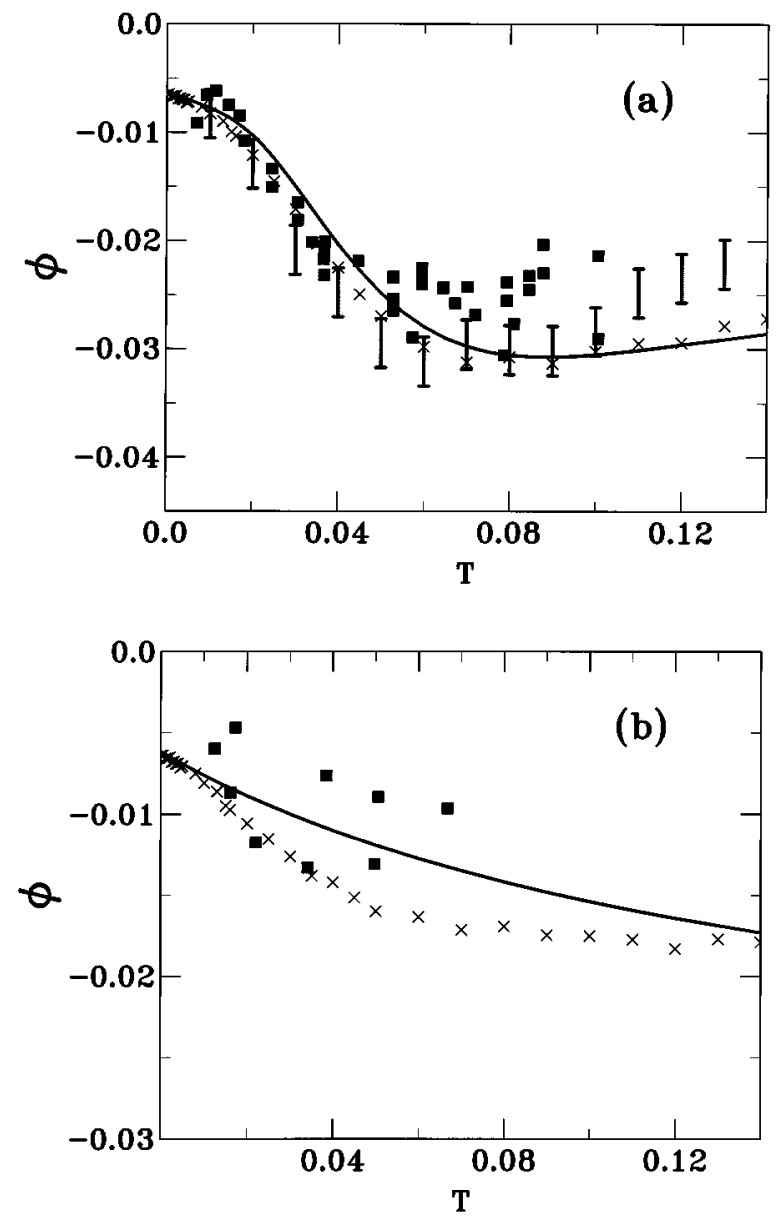

FIG. 8. (a) Dependence on noise intensity $T$ (in units of $\left.\omega_{0}^{4} / \gamma\right)$ of the phase $\phi$ of the response at the frequency $\Omega$ of the driving force, for $\Omega / \omega_{0}=0.415$ and the reduced field amplitude $F=1.76 \times 10^{-2}$ (solid squares). The results are compared to the theory (solid line) for $\phi$ (see text), to the values of $\phi$ obtained from computer simulations of the spectral density of fluctuations (crosses), and to direct computer simulations of $\phi$ for these parameters (bars). (b) As in (a), but without direct computer simulations and with $F=4.4 \times 10^{-3}$, i.e., for a driving force only a quarter as strong, so that $\phi \approx \phi^{(1)}$.

tions were omitted in this case as being too time consuming.) Note that the relatively large scatter of the data arose because the values of $\phi^{(1)}$ far from resonance were extremely small, so that the measurements were necessarily being performed close to the instrumental resolution: the most important point to note is that the results were clearly very different from those of Figs. 7(a) and 8(a).

The dependence on noise intensity of the linear phase shift at a nonresonant frequency is monotonic. However, this dependence is unexpectedly strong for the value of the cubic nonlinearity parameter $\beta=\frac{5}{3}$ used in the experiments. The numerical values of the reduced noise intensity $\gamma T / \omega_{0}^{4}$ for which deviations from (10) become substantial are as small as $2 \times 10^{-2}$. Formally, this is a consequence of large numerical factors in the expression for the correction $\propto \beta^{2}$ in Eq. (10). Intuitively, it can be understood from the shape of the potential of the oscillator: for the value $\beta=\frac{5}{3}$ this potential has already become strongly anharmonic for energies that are much smaller than the characteristic energy value $\omega_{0}^{6} / \beta^{2}$ 


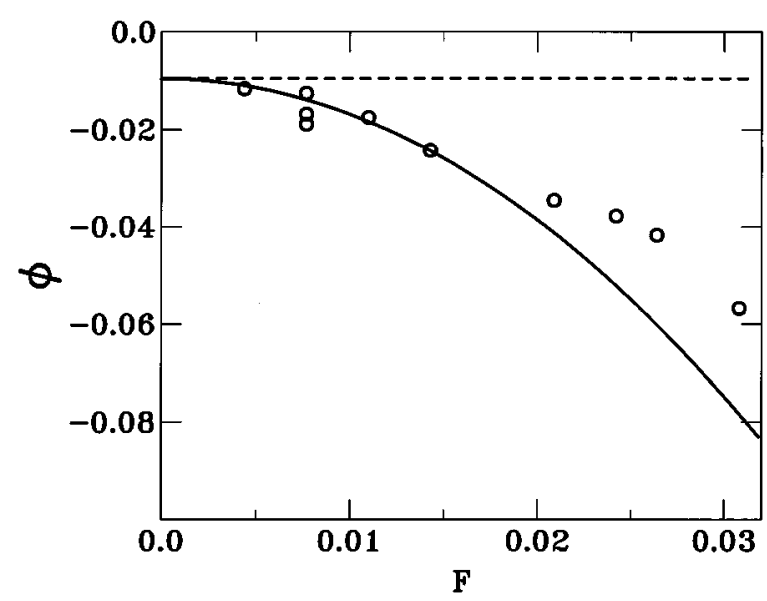

FIG. 9. The dependence of the phase shift $\phi$ on the the reduced field amplitude $F$ of the force for $\Omega / \omega_{0}=0.483$ and noise intensity $\gamma T / \omega_{0}^{4}=0.012$, which corresponds to the absorption maximum at this frequency. Measurements on the analog electronic circuit (circles) are compared with the theory (full curve). The dashed line indicates the position of $\phi^{(1)}$.

used in (3) as the upper limit of noise intensity for which the theory applies.

In Fig. 9 we present the measured and calculated dependences of $\phi$ on the amplitude $F$ of the driving force. It is seen that $\phi$ increases nearly linearly with the squared amplitude of the force, $F^{2}$, as indicated by the theoretical curve. These results provide a clear demonstration that the phenomena we are observing correspond to two-photon absorption [cf. Eq. (13)]. Note, in particular, that the value of the force amplitude used in all experiments (except the linear phase shift measurements) was chosen as the largest amplitude for which the theory was expected to be applicable, and that, for small amplitudes $F, \phi$ approaches the value of the phase shift for linear response $\phi^{(1)}$. This latter feature made it possible to separate the linear and nonlinear contributions to the phase shift, simply by changing the amplitude of the driving force in the experiment.

Finally we note that the theory can immediately be generalized in two ways. First of all, strictly speaking, Eqs. (7) and (8) which we used to obtain the theoretical results and the computer simulation data shown in Figs. 2-8, do not apply away from range (3); and the full theory discussed in Appendix A should then be used. However, in the most interesting range of $T$ where the dependence of the SHS on noise intensity is nonmonotonic, the amplitudes of vibration of the oscillator at the overtones of the eigenfrequencies $\omega(E)$ are small for energies $E \leqslant T$, and the simple theory of Sec. II provides a good approximation. Second, the numerical algorithm [12] used to evaluate the spectral density of fluctuations, and thus the linear susceptibility (9), is limited to resonant frequencies where $Q^{(0)}(\omega)$ has a peak. General analysis of the linear susceptibility requires a complete solution of the appropriately modified Fokker-Planck equation. We have developed the necessary algorithm, and details will be given in a later paper.

\section{CONCLUSIONS}

In conclusion, we have developed a theory of an underdamped nonlinear oscillator driven by a field at a subhar- monic frequency $\Omega$, and carried out a detailed experimental investigation of the oscillator dynamics. The theory applies for an arbitrary nonlinearity of the oscillator. For moderately strong noise, the resonant nonlinear response of the oscillator has been described in terms of a linear susceptibility. The results describe the spectra of nonlinear absorption (the classical analog of two-photon absorption) and the signal at the second harmonic as functions of the fluctuation intensity. We have been able to predict, and measure, a strong noiseinduced amplification of resonant SHG and of TPA.

We have shown that both the signal at the second harmonic, and the nonlinear absorption, display peaks as a function of the noise intensity $T$. The peaks are sharp if $2 \Omega$ lies close to the frequency $\omega_{0}$ of small-amplitude vibrations of the oscillator. We have shown that peaks can also occur if $2 \Omega$ is close to the extremal value $\omega_{e}$ of the oscillator's eigenfrequency $\omega(E)$. The theory is in good qualitative and quantitative agreement with experiment. The results also agree well with those obtained from computer simulations of a nonlinear oscillator in the absence of periodic driving. The effects considered may be of importance in connection with a number of applications, from nonlinear optics and twophoton spectroscopy to nonlinear response of underdamped superconducting quantum interference devices.

Our results demonstrate that a simple theoretical model can provide physical insight into the interrelation between the linear and nonlinear responses of the oscillator and reveal the connection between two-photon absorption in underdamped systems and stochastic resonance.

\section{ACKNOWLEDGMENTS}

The work was supported by the Center for Fundamental Material Research at MSU, the Engineering and Physical Sciences Research Council (UK), the European Community, the Royal Society of London, the Gosstandart of Russia and the International Science Foundation under Grant Nos. N62000 and N62300. DGL gratefully acknowledges the extremely warm hospitality of Lancaster University.

\section{APPENDIX A: NONLINEAR RESPONSE OF AN OSCILLATOR IN THE LIMIT OF SMALL DAMPING}

In this appendix we consider the nonlinear response of an underdamped oscillator in the general case in which the noise intensity is not limited to moderately small values as specified by (3). For an oscillator performing Brownian motion the analysis may be done using the Fokker-Planck equation (FPE) for the probability density $w\left(I, \phi, t \mid I_{0}, \phi_{0}, t_{0}\right)$ of a transition from a state with a given action $I_{0}$ and phase $\phi_{0}$ occupied at initial time $t_{0}$ to a state $(I, \phi)$ at time $t$. If the oscillator is described by Eq. (1), the FPE may be written in the form [32]

$$
\frac{\partial w}{\partial t}=-\omega(E) \frac{\partial w}{\partial \phi}-F \cos \Omega t \frac{\partial w}{\partial p}+\Gamma \hat{L} w
$$

Here $\hat{L}$ is an operator that describes the dissipation and diffusion terms in the FPE. In the absence of forcing it results in 
$w\left(I, \phi, t \mid I_{0}, \phi_{0}, t_{0}\right)$ approaching the stationary distribution $w_{\mathrm{st}}^{(0)}(I, \phi)$ as the time interval $t-t_{0} \rightarrow \infty$. For thermal equilibrium systems,

$$
\begin{gathered}
w_{\mathrm{st}}^{(0)}(I, \phi)=Z^{-1} \exp [-E(I) / T] \\
I=\int_{0}^{E(I)} \omega^{-1}(E) d E, \quad Z=2 \pi \int d I \exp [-E(I) / T] .
\end{gathered}
$$

The coordinate and momentum of the oscillator $q$ and $p$ are periodic functions of the phase $\phi$,

$$
\begin{gathered}
p \equiv p(I, \phi) \equiv p(I, \phi+2 \pi), \\
q \equiv q(I, \phi) \equiv q(I, \phi+2 \pi)=\sum_{n} a_{n}(I) e^{i n \phi}, \\
p=\omega(E) \frac{\partial q}{\partial \phi}, \quad \frac{\partial p}{\partial I} \frac{\partial q}{\partial \phi}-\frac{\partial q}{\partial I} \frac{\partial p}{\partial \phi}=1 .
\end{gathered}
$$

The procedure for finding the Fourier components of the coordinate $a_{n}(I) \equiv a_{n}^{*}(I)$ is standard. It becomes particularly simple for an oscillator with a potential of the form of a fourth-order polynomial in $q$ as in Eq. (1) (cf. [12]), since in this case the solution can be written explicitly in terms of elliptic functions.

We will be seeking the asymptotic distribution of a periodically driven oscillator $w_{\text {as }}(I, \phi, t)$. This distribution is periodic in time, with period $2 \pi / \Omega$; it too is periodic in $\phi$,

$$
\begin{gathered}
w_{\text {as }}(I, \phi, t)=\sum_{n} w_{n}(I, t) e^{i n \phi}, \\
w_{n}(I, t+2 \pi / \Omega)=w_{n}(I, t), \\
\langle q(t)\rangle=2 \pi \sum_{n} \int d I a_{-n} w_{n}(I, t) .
\end{gathered}
$$

It is known from the theory of the linear response of a nonlinear oscillator [14] that the shape of the distribution (A4) is determined by the relation between the fluctuation-induced smearing $\delta \omega$ of the oscillator frequency $\omega(E)$ and the oscillator damping $\Gamma$. For $\delta \omega \gg \Gamma$ damping affects resonant linear response primarily through the form of the stationary distribution in the absence of driving (A2). This is also true for a resonant nonlinear response. Indeed, a nonlinear oscillator has a continuous frequency spectrum, of width $\delta \omega \sim|\omega(T)-\omega(0)|$, and the additional frequency smearing due to the lifetime being finite may be neglected when this lifetime is large, i.e., when $\Gamma \ll \delta \omega$ [the case where $\omega(E)$ has an extremum $\omega_{e}$ and $2 \Omega \approx \omega_{e}$ requires special consideration; the corresponding analysis for the case of resonant onephoton absorption where $\Omega \approx \omega_{e}$ was done in [25]].

For comparatively weak driving, the solution of Eq. (A1) for the functions $w_{n}(I, \phi)$ can be obtained by direct perturbation theory in the force amplitude $F$. To first order in $F$, neglecting dissipation, we have

$$
\begin{gathered}
w_{n}^{(1)}(I, t)=F\left[W_{n}^{(1)}(I, \Omega) e^{i \Omega t}+W_{n}^{(1)}(I,-\Omega) e^{-i \Omega t}\right], \\
W_{n}^{(1)}(I, \Omega) \approx \frac{1}{2 T} \frac{a_{n}(I) n \omega(E)}{n \omega(E)+\Omega} w_{\mathrm{st}}^{(0)}
\end{gathered}
$$

We notice that, in the case of driving at a subharmonic frequency as considered in the present paper, the denominators in (A5) are nonresonant. However Eq. (A5) also describes, for $\delta \omega \gg \Gamma$, the resonant linear response to driving at frequencies $\Omega$ which lie within the "band" of the oscillator frequencies $\omega(E)$ with $E \lesssim T$, or within the corresponding bands at the overtones of $\omega(E)$. The singularity in the denominator can be dealt with in a standard way by making the replacement

$$
n \omega(E) \rightarrow n \omega(E)-i \delta, \quad \delta \rightarrow 0^{+},
$$

which allows for the decay of correlations in the system.

To second order in $F$, the stationary distribution $w_{\text {st }}(I, \phi, t)$ contains terms oscillating at frequency $2 \Omega$, and it also contains a nonresonant time-independent term $\propto F^{2}$,

$$
\begin{aligned}
w_{n}^{(2)}(I, t)= & F^{2}\left[W_{n}^{(2)}(I, 2 \Omega) e^{2 i \Omega t}+W_{n}^{(2)}(I,-2 \Omega) e^{-2 i \Omega t}\right. \\
& \left.+\widetilde{W}_{n}^{(2)}(I, 0)\right]
\end{aligned}
$$

where

$$
\begin{aligned}
W_{n}^{(2)}(I, 2 \Omega) \approx & \frac{1}{2}[2 \Omega+n \omega(E)]^{-1} \sum_{m}\left[(n-m) \frac{d a_{m}}{d I}\right. \\
& \left.\times W_{n-m}^{(1)}(I, \Omega)-m a_{m}(I) \frac{\partial W_{n-m}^{(1)}(I, \Omega)}{\partial I}\right] .
\end{aligned}
$$

It is clear from (A7) that, if the frequency $2 \Omega$ lies within the band of the eigenfrequencies $\omega(E)$ with $E \leqslant T$, the terms $W_{-1}^{(2)}(I, 2 \Omega), W_{1}^{(2)}(I,-2 \Omega)$ contain resonant denominators. Equations (A4) and (A7) thus provide a solution to the problem of resonant enhancement of the signal at the second harmonic of the driving force, i.e., of resonant secondharmonic generation.

With Eqs. (5) and (A3) taken into account, the coefficient of two-photon absorption can be written as

$$
\begin{aligned}
\kappa^{(2)}= & i \pi F^{2} \int d I \sum_{n} n \omega(E) a_{-n}(I)\left[W_{n}^{(3)}(I,-\Omega)\right. \\
& \left.+W_{n}^{(3)}(I, \Omega)\right],
\end{aligned}
$$

where $W_{n}^{(3)}$ is the coefficient in the expression for the third order (in $F$ ) correction to the stationary distribution $w_{\text {st }}(\mathrm{A} 4)$,

$$
\begin{aligned}
w_{n}^{(3)}(I, t)= & F^{3}\left[W_{n}^{(3)}(I, \Omega) e^{i \Omega t}+W_{n}^{(3)}(I,-\Omega) e^{-i \Omega t}\right. \\
& \left.+\widetilde{W}_{n}^{(3)}(I, 3 \Omega) e^{3 i \Omega t}+\widetilde{W}_{n}^{(3)}(I,-3 \Omega) e^{-3 i \Omega t}\right] .
\end{aligned}
$$

The functions $W_{n}^{(3)}$ can be expressed in terms of the lowerorder terms $W_{n}^{(2)}, \widetilde{W}_{n}^{(2)}$ in precisely the same way as the latter have been expressed in terms of $W_{n}^{(1)} \mathrm{cf}$. (A7).

It is clear that if, in the expressions for the $W_{n}$, the real parts of the frequency-dependent denominators 
$n \omega(E) \pm n^{\prime} \Omega$ did not become equal to zero for some $E$, then there would hold a relation $W_{n}^{(3)}(I,-\Omega)=W_{-n}^{(3)}(I, \Omega)$, and the absorption coefficient (A8) would be equal to zero. The absorption is due to resonant processes in which the oscillator is excited by the field; in the present case these are twophoton processes. The corresponding resonant denominators are of the form of $2 \Omega-\omega(E)$. They enter expression (A7) for $W_{n}^{(2)}(I,-\Omega)$, and thus they enter the terms in $W_{n}^{(3)}(I, \pm \Omega)$ that are proportional to $W_{n}^{(2)}(I, \pm \Omega)$ or to the derivative $\partial W_{n}^{(2)}(I, \pm \Omega) / \partial I$. It is straightforward to implement on the computer the corresponding algorithm for evaluation of the coefficient of two-photon absorption.

\section{APPENDIX B: NOISE-INDUCED CORRECTIONS TO THE LINEAR NONRESONANT SUSCEPTIBILITY}

Suppose we have a Brownian particle, with the equation of motion

$$
\begin{gathered}
\ddot{q}+2 \Gamma \dot{q}+\omega_{0}^{2} q+\beta q^{2}+\gamma q^{3}=f(t), \\
\left\langle f(t) f\left(t^{\prime}\right)\right\rangle=4 \Gamma T \delta\left(t-t^{\prime}\right),
\end{gathered}
$$

and that the damping and temperature are both small, $\Gamma \ll \omega_{0},|\gamma| T / \omega_{0}^{4} \ll 1$, and $\beta^{2} T / \omega_{0}^{6} \ll 1$. We are interested in the linear response of the system to a nonresonant force. In the harmonic approximation the susceptibility

$$
\chi^{(0)}(\Omega)=\left(\omega_{0}^{2}-\Omega^{2}-2 i \Gamma \Omega\right)^{-1} .
$$

To allow for nonlinear corrections to first order in $T$, it is convenient to start from the corresponding Fokker-Plank equation

$$
\begin{gathered}
\frac{\partial w}{\partial t}=-\frac{\partial(p w)}{\partial q}-\frac{\partial}{\partial p}\left\{\left[-2 \Gamma p-U^{\prime}(q)\right] w\right\} \\
+2 \Gamma T \frac{\partial^{2} w}{\partial p^{2}}-F e^{-i \Omega t} \frac{\partial w}{\partial p} \\
U^{\prime}(q)=\omega_{0}^{2} q+\beta q^{2}+\gamma q^{3} .
\end{gathered}
$$

In linear response theory, we seek a solution in the form

$$
w=w^{(0)}+F e^{-i \Omega t} w^{(1)}, \quad w^{(0)}=Z^{-1} \exp \left(-\frac{p^{2}}{2 T}-\frac{U(q)}{T}\right),
$$

and assume that the correction term $w^{(1)}$ is independent of time and of field amplitude $F$. The equation for $w^{(1)}$ is of the form

$$
\begin{gathered}
-i \Omega w^{(1)}+\frac{\partial\left(p w^{(1)}\right)}{\partial q}+\frac{\partial}{\partial p}\left\{\left[-2 \Gamma p-U^{\prime}(q)\right] w^{(1)}\right\} \\
-2 \Gamma T \frac{\partial^{2} w^{(1)}}{\partial p^{2}}=\frac{p}{T} w^{(0)}
\end{gathered}
$$

The asymptotic solution of this equation at low temperatures, $T \rightarrow 0$, may be obtained by applying the method of moments. We consider the quantities

$$
R_{m n} \equiv \int d q d p q^{m} p^{n} w^{(1)} .
$$

The susceptibility is

$$
\chi(\Omega)=R_{10} .
$$

From (B5) we obtain a set of equation:

$$
\begin{aligned}
(- & i \Omega+2 \Gamma n) R_{m n}-m R_{m-1 n+1}+n\left(\omega_{0}^{2} R_{m+1 n-1}\right. \\
& \left.+\beta R_{m+2 n-1}+\gamma R_{m+3 n-1}\right)-2 \Gamma \operatorname{Tn}(n-1) R_{m n-2} \\
= & T^{-1}\left\langle q^{m} p^{n+1}\right\rangle_{0} .
\end{aligned}
$$

The averaging on the right-hand side of (B8) is performed with the equilibrium distribution $w^{(0)}$. We have

$$
\left\langle q^{m} p^{n}\right\rangle_{0}=\left\langle q^{m}\right\rangle_{0}\left\langle p^{n}\right\rangle_{0}, \quad\left\langle p^{2 n}\right\rangle_{0}=\frac{1}{\pi^{1 / 2}}(2 T)^{n}\left(n-\frac{1}{2}\right) !
$$

In particular,

$$
\left\langle p^{2}\right\rangle_{0}=T, \quad\left\langle p^{4}\right\rangle_{0}=3 T^{2} .
$$

To first order in $\beta$,

$$
\begin{gathered}
\left\langle q^{2 m}\right\rangle_{0} \approx \frac{1}{\pi^{1 / 2}}\left(\frac{2 T}{\omega_{0}^{2}}\right)^{m}\left(m-\frac{1}{2}\right) !, \\
\left\langle q^{2 m+1}\right\rangle_{0} \approx-\frac{\beta}{3 \pi^{1 / 2} T}\left(\frac{2 T}{\omega_{0}^{2}}\right)^{(m+2)}\left(m+\frac{3}{2}\right) ! .
\end{gathered}
$$

The set of algebraic equations (B8) should be solved for the conditions $R_{m n}=0$ for negative $m$ or $n$. It is seen from (B8) that, if we are interested in the terms linear in temperature, it suffices to restrict ourselves to $R_{m n}$ with $m+n \leqslant 3$, and to set

$$
R_{m n}=0 \text { for } m+n>3 .
$$

This reduces (B8) to a set of nine equations. They can be easily solved, and the resulting expression for the the susceptiblity takes the form of Eq. (10) in the text.
[1] L. D. Landau and E. M. Lifshitz, Mechanics (Pergamon, London 1976).

[2] Y. R. Shen, The Principles of Nonlinear Optics (Wiley, New York, 1984).

[3] M. A. Ivanov, L. B. Kvashnina, and M. A. Krivoglaz, Fiz. Tverd Tela (Leningrad) 7, 2047 (1965) [Sov. Phys. Solid State 7, 1652 (1965)].

[4] R. J. Elliott, W. Hayes, G. D. Jones, H. F. Macdonald, and C. T. Sennett, Proc. R. Soc. London A Ser. 289, 1 (1965).
[5] J. B. Morton and S. Corrsin, J. Stat. Phys. 2, 153 (1970).

[6] M. A. Krivoglaz and I. P. Pinkevich, Ukr. Fiz. Zh. 15, 2039 (1970); Y. Onodera, Prog. Theor. Phys. 44, 1477 (1970).

[7] M. I. Dykman and M. A. Krivoglaz, Phys. Status Solidi B 48, 497 (1971); Physica A 104, 495 (1980).

[8] M. Bixon and R. Zwanzig, J. Stat. Phys. 3, 245 (1971); K. Sture, J. Nordholm, and R. Zwanzig, ibid. 11, 143 (1974); R. F. Rodriguez and N. G. van Kampen, Physica A 85, 347 (1976). 
[9] A. B. Budgor, K. Lindenberg, and K. E. Shuler, J. Stat. Phys. 15, 375 (1976); A. R. Bulsara, K. Lindenberg, and K. E. Shuler, ibid. 27, 787 (1982).

[10] W. Renz, Z. Phys. B 59, 91 (1985); L. Fronzoni, P. Grigolini, R. Mannella, and B. Zambon, J. Stat. Phys. 41, 553 (1985); Phys. Rev. A 34, 3293 (1986); W. Renz and F. Marchesoni, Phys. Lett. 112A, 124 (1985).

[11] M. I. Dykman, R. Mannella, P. V. E. McClintock, F. Moss, and S. M. Soskin, Phys. Rev. A 37, 1303 (1988).

[12] M. I. Dykman, R. Mannella, P. V. E. McClintock, S. M. Soskin and N. G. Stocks, Phys. Rev. A 42, 7041 (1990).

[13] V. I. Mel'nikov, J. Stat. Phys. 70, 77 (1993).

[14] M. I. Dykman and M. A. Krivoglaz, in Soviet Physics Reviews, edited by I. M. Khalatnikov (Harwood, New York, 1984), Vol 5, p. 265; M. I. Dykman and P. V. E. McClintock, Physica D 58, 10 (1992).

[15] A. S. Barker Jr. and A. J. Sievers, Rev. Mod. Phys. 47, Suppl. No 2, S1 (1975).

[16] J.A. Goldstone and E. Garmire, Phys. Rev. Lett. 53, 910 (1984).

[17] H. Carmichael, An Open System Approach to Quantum Optics (Springer, Berlin 1993).

[18] G. Gabrielse, H. Dehmelt, and W. Kells, Phys. Rev. Lett. 54, 537 (1985).

[19] W.F. Vinen, N.J. Appleyard, L. Skrbek and P.K.H. Sommerfeld, Physica B 197, 360 (1994).

[20] R. Superfine, J.Y. Huang, and Y.R. Shen, Phys. Rev. Lett. 66, 1066 (1991).

[21] Q. Du, R. Superfine, E. Freysz, and Y.R. Shen, Phys. Rev. Lett. 70, 2313 (1993).

[22] D. von der Linde, T. Engers, G. Jenke, P Agostini, G. Grillon, E. Nibbering, A. Mysyrowicz, and A. Antonetti, Phys. Rev. A 52, R25 (1995).
[23] Stochastic Resonance in Physics and Biology, special issue of J. Stat. Phys. 70 (1/2), (1993) edited by F. Moss, A. Bulsara, and M. F. Shlesinger; Fluctuations in Physics and Biology: Stochastic Resonance, Signal Processing and Related Phenomena, special issue of Nuovo Cimento D 17(7/8), edited by A. Bulsara, S. Chillemi, L. Kiss, P. V. E. McClintock, R. Mannella, F. Marchesoni, K. Nicolis, and K. Wiesenfeld (1995).

[24] N. G. Stocks, N. D. Stein, S. M. Soskin, and P. V. E. McClintock, J. Phys. A 25, L1119 (1992); N. G. Stocks, N. D. Stein, and P. V. E. McClintock, ibid. 26, L385 (1993).

[25] S. M. Soskin, Physica A 155, 401 (1989); 180, 386 (1992); N. G. Stocks, P. V. E. McClintock, and S. M. Soskin, Europhys. Lett. 21, 395 (1993).

[26] L. D. Landau and E. M. Lifshitz, Statistical Physics, 3rd ed. (Pergamon, New York, 1980), p.1, revised by E. M. Lifshitz and L. P. Pitaevskii.

[27] L. Fronzoni in Noise in Nonlinear Dynamical Systems, edited by F. Moss and P. V. E. McClintock (Cambridge University Press, Cambridge, England, 1989), Vol. 3, p. 222; P. V. E. McClintock and F. Moss, ibid., p. 243.

[28] P. V. E. McClintock, S. M. Soskin, N. D. Stein, and N. G. Stocks, Phys. Rev. E 48, 147 (1993).

[29] M. I. Dykman, D. G. Luchinsky, R. Mannella, P. V. E. McClintock, N. D. Stein, and N. G. Stocks, Adv. Chem. Phys. LXXXV, 265 (1994).

[30] H. Yoshida, Phys. Lett. A 150, 262 (1990).

[31] M. I. Dykman, D. G. Luchinsky, R. Mannella, P. V. E. McClintock, H. E. Short, N. D. Stein, and N. G. Stocks, Phys. Lett. 193A, 61 (1994).

[32] H. Risken, The Fokker-Planck Equation, 2nd ed. (Springer, Berlin, 1989). 